\title{
The Remnant and Origin of the Historical Supernova 1181 AD
}

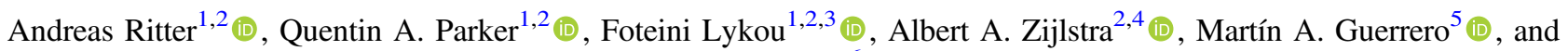 \\ Pascal Le Dû́ ${ }^{6}$ (D) \\ ${ }^{1}$ Department of Physics, The University of Hong Kong, Chong Yuet Ming Physics Building, Pokfulam Road, Hong Kong; quentinp@ @ku.hk \\ ${ }^{2}$ Laboratory for Space Research, The University of Hong Kong, 405B Cyberport 4, 100 Cyberport Road, Cyberport, Hong Kong \\ ${ }^{3}$ Konkoly Observatory, Research Centre for Astronomy and Earth Sciences, Konkoly-Thege Miklós út 15-17, 1121 Budapest, Hungary \\ ${ }_{5}^{4}$ Jodrell Bank Centre for Astrophysics, The University of Manchester, Oxford Road, Manchester M13 9PL, UK \\ ${ }^{5}$ Instituto de Astrofísica de Andalucía (IAA-CSIC), Glorieta de la Astronomía S/N, E-18008 Granada, Spain \\ ${ }^{6}$ Kermerrien Observatory, F-29840 Porspoder, France \\ Received 2021 May 18; revised 2021 August 30; accepted 2021 August 31; published 2021 September 15
}

\begin{abstract}
The guest star of AD 1181 is the only historical supernova of the past millennium that is without a definite counterpart. The previously proposed association with supernova remnant G130.7+3.1 (3C 58) is in strong doubt because of the inferred age of this remnant. Here we report a new identification of SN 1181 with our codiscovery of the hottest known Wolf-Rayet star of the oxygen sequence (IRAS 00500+6713 or 2MASS J00531123+6730023, here named by us as "Parker's star") and its surrounding nebula Pa 30. Our spectroscopy of the nebula shows a fast shock with extreme velocities of $\approx 1100 \mathrm{~km} \mathrm{~s}^{-1}$. The derived expansion age of the nebula implies an explosive event $\approx 1000 \mathrm{yr}$ ago that agrees with the 1181 event. The on-sky location also fits the historical Chinese and Japanese reports of SN 1181 to within 3.5. Pa 30 and Parker's star have previously been proposed to be the result of a double-degenerate merger, leading to a rare Type Iax supernova. The likely historical magnitude and the distance suggest the event was subluminous for normal supernova. This agrees with the proposed Type Iax association that would also be only the second of its kind in the Galaxy. Taken together, the age, location, event magnitude, and duration elevate Pa 30 to prime position as the counterpart of SN 1181. This source is the only Type Iax supernova where detailed studies of the remnant star and nebula are possible. It provides strong observational support for the double-degenerate merger scenario for Type Iax supernovae.
\end{abstract}

Unified Astronomy Thesaurus concepts: Supernovae (1668); Supernova remnants (1667); Wolf-Rayet stars (1806)

\section{Introduction}

Only nine historically recorded supernova (SN) explosions are known in the Galaxy (Green 2002). In only five cases has the remnant of the supernova been identified. For the other cases, the remnant is not known with certainty. The remnant is crucial for identifying the type of supernova, while the known time of the explosion and duration constrain the models of the evolution of the remnant.

Here, we consider the historical "Guest Star" of $1181 \mathrm{AD}$ recorded by Chinese and Japanese astronomers (Hoffmann et al. 2020). It remained visible for 185 days from 1181 August 6 to 1182 February $6 \mathrm{AD}$ (Hsi 1957). The supernova remnant (SNR) or pulsar wind nebula G130.7+3.1 (3C 58 hereafter) is located in its vicinity and has, until now, been considered by some authors (e.g., Stephenson \& Green 1999; Green 2002; Kothes 2013) as linked with SN 1181. However, a precise estimate of the expansion age of this nebula of $7000 \mathrm{yr}$, based on radio observations over $20 \mathrm{yr}$ (Bietenholz 2006), and the spin-down age of the pulsar (5400 yr; Chevalier 2004) have put the association in serious doubt, although not completely excluding it (Kothes 2013). A strong argument for this association was that until now there was no other viable candidate known for the remnant. This left SN 1181 as the youngest Galactic supernova without a firmly confirmed remnant. We propose that the recently discovered nebula Pa 30 and its extreme central star (Gvaramadze et al. 2019)

Original content from this work may be used under the terms of the Creative Commons Attribution 4.0 licence. Any further distribution of this work must maintain attribution to the author(s) and the title of the work, journal citation and DOI. are in fact the remnant and residual core of the $1181 \mathrm{AD}$ explosion.

The nebula $\mathrm{Pa} 30$ was discovered on 2013 August 25 from the Wide-field Infrared Survey Explorer (WISE) mid-IR image archive (Cutri et al. 2012) by Dana Patchick (Kronberger et al. 2016) from our affiliated "Deep Sky Hunters" (DSH) amateur astronomer group, and is included in the HASH PN database ${ }^{7}$ (Parker et al. 2016) as Pa 30. The WISE W3 $(11 \mu \mathrm{m})$ band shows a disk-like nebula, whereas the dominant shape in the W4 $(22 \mu \mathrm{m})$ band is donut-like inside a much fainter halo. Narrowband [O III] imaging observations obtained by the DSH group on the $2.1 \mathrm{~m}$ Kitt Peak National Observatory (KPNO) telescope in 2013 September indicate a very faint, diffuse, circular emission feature. The nebula is a strong source of diffuse X-ray emission (Oskinova et al. 2020). Multiwavelength images of the nebula at the same angular scale are shown in Figure 1. The bright central star (CS) is both hydrogen-poor and helium-poor, and has a unique emissionline spectrum as shown by Gvaramadze et al. (2019) and from our earlier, independent spectroscopy (see below).

On careful scrutiny both sets of our spectroscopic observations revealed two sets of faint optical emission lines species in the nebula: the [S II] 6716 and $6731 \AA$ doublet, and the [Ar III] $7136 \AA$ line. The well-resolved [S II] doublet in particular shows two sets of lines due to extreme velocity expansion of the nebula gas, with radial velocities of up to $\pm v_{\text {rad }} \approx 1100 \pm 100 \mathrm{~km} \mathrm{~s}^{-1}$ (Figure 2). The observed velocity structure is approximately constant up to $50^{\prime \prime}$ from the CS, followed by a sharp decline to

\footnotetext{
http://hashpn.space
} 

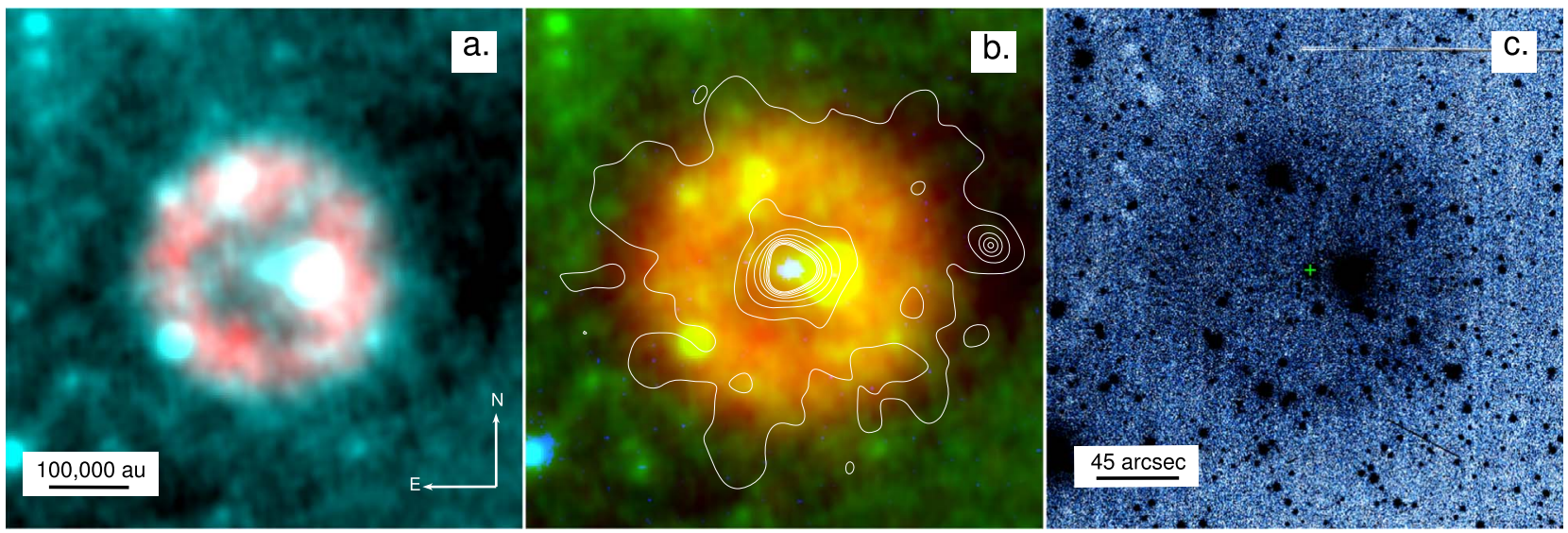

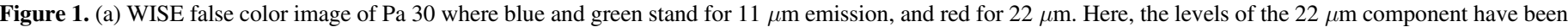

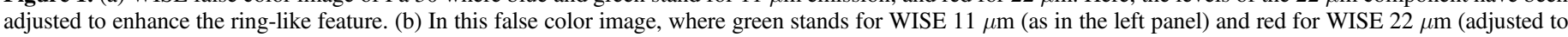

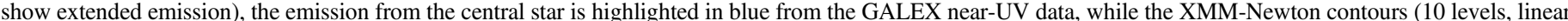

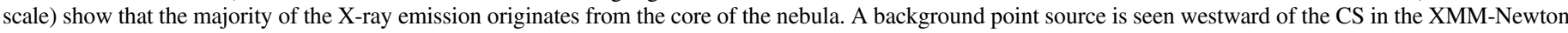

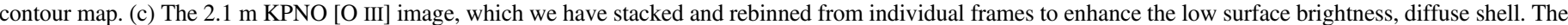

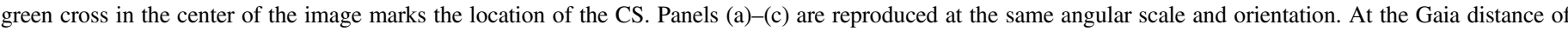
$\mathrm{Pa} 30$ of $2.30 \pm 0.14 \mathrm{kpc}$, an angular scale of $45^{\prime \prime}$ translates to about 100,000 au.

systemic velocity at $\approx 100^{\prime \prime}$. We also extracted the star's GranTeCan (GTC) spectrum after it was first brought to our attention by Pascal Le Dû, an amateur collaborator of the corresponding author. We recognized its extreme and unique nature (e.g., see Gvaramadze et al. 2019), believing we were the first group to do so. We thereafter referred to IRAS $00500+6713$ as "Parker's star."

Assuming that the angular radius of the shell is $100^{\prime \prime} \pm 10^{\prime \prime}$ and taking the latest Gaia distance of $2.30 \pm 0.14 \mathrm{kpc}$ (Bailer-Jones et al. 2021), the highest-velocity shell extends to a total diameter of $2.2 \pm 0.4 \mathrm{pc}$. If we further assume that the $1100 \mathrm{~km} \mathrm{~s}^{-1}$ gas is expanding at a constant velocity, and that the uncertainty on the expansion velocity is $10 \%$, the highest-velocity shell has a kinematic age of $990_{-220}^{+280} \mathrm{yr}$.

After free expansion, which usually lasts for a few hundred years, typical SNRs enter the Sedov phase and slow down due to sweeping up the Interstellar and Circumstellar Medium (ISM/CSM) once the swept-up mass is similar to the mass of the ejecta. In this particular case, however, there are two reasons why the shell could still be in the free expansion phase. First, the presence of an outer shell indicates that the ISM/ CSM has already been swept up by some previous event, allowing for free expansion of the highest-velocity shell until now. The second reason is that, if Parker's star and $\mathrm{Pa} 30$ are indeed the remnant and ejecta of two merging white dwarfs (WDs), as suggested by Gvaramadze et al. (2019) and Oskinova et al. (2020), the mass-loss rate of WDs can be effectively ignored. Hence, the density of the CSM would remain very low. If on the other hand the shell was indeed in the Sedov phase it would have to be significantly younger and our age estimate would be an upper limit. In that case, however, given the advances in astronomy over the past $1000 \mathrm{yr}$, especially in China, the SN itself should have been detected by astronomers more recently. This is not the case.

Oskinova et al. (2020) estimated an age range between 350 and $1100 \mathrm{yr}$ based on the inner ring-like shell and the wind ram pressure and thermal pressure. These values support the identification of the nebula as an SNR. The kinematic age of Pa 30 agrees well with SN 1181 recorded 840 yr ago and provides a strong temporal argument for association. The second argument for the association comes from the position concordance. Of the recorded "guest stars" listed in Hoffmann et al. (2020), SN 1181 is the only one that matches the estimated explosion date and the location of $\mathrm{Pa} 30$ to within the errors. Averaging the five reported positions for this historical SN (Hoffmann et al. 2020) and transforming the average to J2000, the separation on the sky between the recorded position and the position of Parker's star is only 3.5 (see Table 1), well within the uncertainties. The Gaia proper motion for the star of 2.7 mas $^{-1} r^{-1}$ would only shift it by $\sim 3^{\prime \prime}$ in the intervening period. The only other viable alternative (and indeed the previously favored association; Kothes 2013) is SNR 3C 58 at $\sim 4.5$ away from the SN 1181 position.

Figure 3 shows the best estimated general location of SN 1181. The two candidates $\mathrm{Pa} 30$ and 3C 58 are indicated, as is the best estimate for the location of SN 1181 with a $5^{\circ}$ radius error circle. The $\mathrm{SN}$ was reported to have occurred near Wangliang in Huagai, invading Chuanshe (Hsi 1957; spelling adopted from Hoffmann et al. 2020). These Chinese asterisms are shown by the three red-lined constellations, as taken from Hoffmann et al. (2020). The location of Chuanshe is not known with full certainty: the dashed line shows the location according to Stephenson \& Green (2002). The text suggests that SN 1181 should be between the two constellations. Pa 30 fits this well. The alternative, 3C 58, is located in or south of Chuanshe, not obviously associated with Huagai. We also note that the location of 3C 58 would have been more easily described as close to the bright (third magnitude) star $\varepsilon$ Cas but it was not.

The magnitude of SN 1181 is not known with certainty, but SN 1181 was compared in the manuscripts to Saturn. With the reasonable assumption that this refers to its brightness, then the magnitude at peak may have been around $m_{v} \sim-0.5$ to +1.0 . This agrees with the fact it was obvious enough to be picked up in China and Japan, who at the time had well-developed astronomical capacity, but was missed in others (e.g., Korea and Europe). For an assumed distance of $2.30 \pm 0.14 \mathrm{kpc}$ (BailerJones et al. 2021) and an extinction of $A_{V}=2.4$ mag (see below), the absolute magnitude of SN 1181 becomes $m_{v} \sim-14$ to -12.5 . This is significantly subluminous for a typical supernova but is within the range for Type Iax events (see below). The object also 


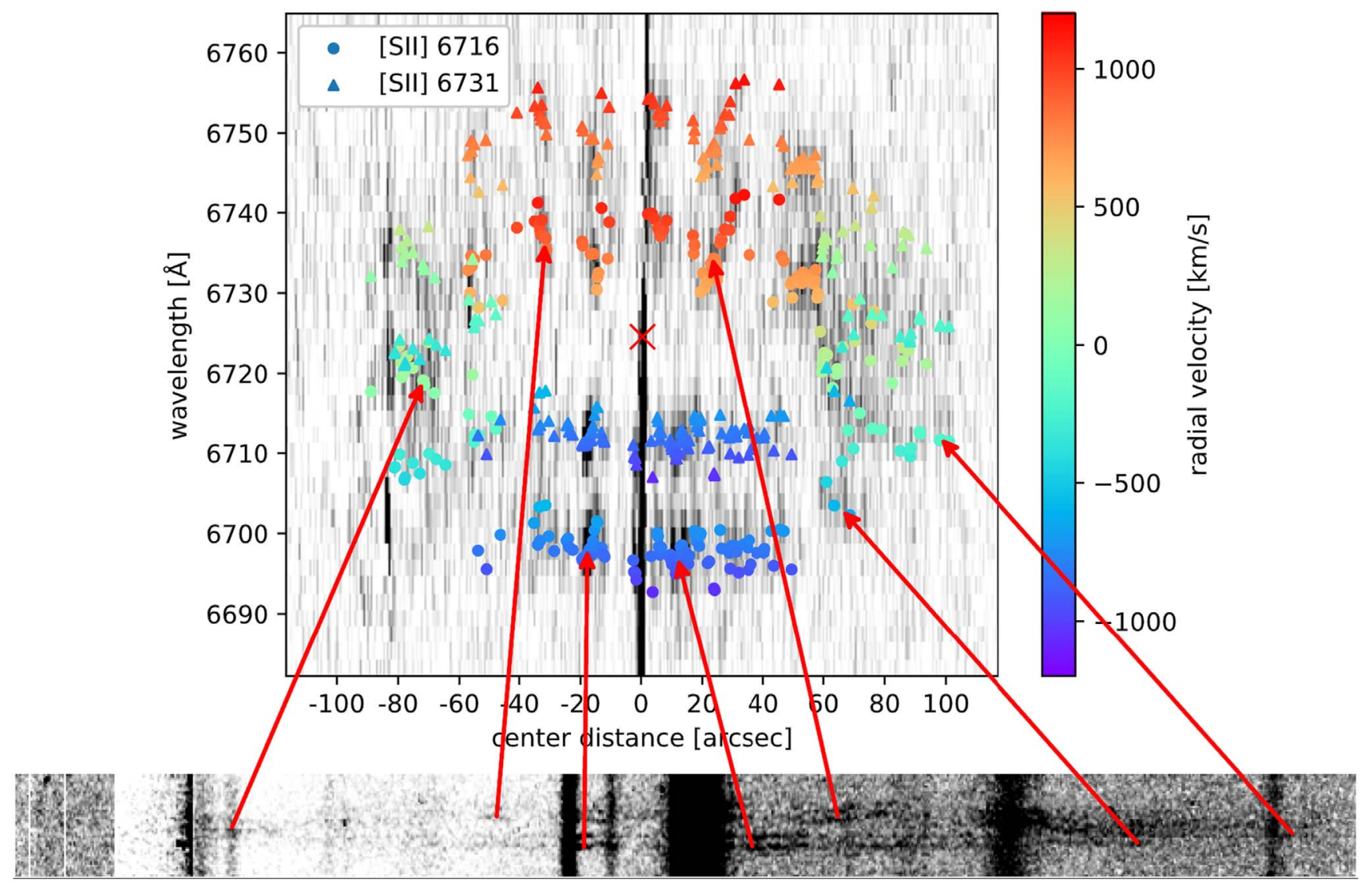

Figure 2. Upper panel: GTC/OSIRIS position-velocity diagram of the [S II] doublet lines (circles: $6716 \AA$, triangles: $6731 \AA$; grayscale inverted and in arbitrary data units) along the slit, overplotted with the measured line positions, and color-coded to the radial velocity for each measured line. Left is NNE of the CS, right is SSW. The position of the CS is marked with a red cross. Spectral features have been enhanced by subtracting the mean of each spectrum above and below the [S II] lines. The image shows sharp velocity variations with increasing distance from the CS at $\approx-20^{\prime \prime},-40^{\prime \prime}$, and $+25^{\prime \prime}$ where the two sets of [S II] doublet lines are also much brighter than for the rest of the long-slit spectrum, indicating strong shock excitation both for the near as well as the far side of the nebula. Lower panel: cutout of the original 2D spectrum (also inverted) showing the same area as the upper panel, enhanced in contrast to make the two sets of [S II] lines more easily visible. The central star is located in the center. The lack of correspondence between the grayscale in the two panels is due to the subtracting the mean of each spectrum above and below the observed [S II] lines in the upper one.

Table 1

Coordinates (J2000)

\begin{tabular}{|c|c|c|c|c|}
\hline & R.A. & Decl. & $\Delta(\mathrm{Pa} 30)$ & $\Delta(3 \mathrm{C} 58)$ \\
\hline \multicolumn{5}{|c|}{ SN 1181 coordinates } \\
\hline Stephenson & 01:33:30 & $+65: 15: 23$ & 4.6 & 3.4 \\
\hline Hsi & $01: 43: 53$ & $+70: 15: 05$ & 5.3 & 5.8 \\
\hline Psovskii & 01:03:12 & $+65: 16: 03$ & 2.4 & 6.6 \\
\hline $\mathrm{Xi}$ & 01:33:30 & $+65: 15: 23$ & 4.6 & 3.4 \\
\hline Hoffmann & $01: 31: 14$ & $+70: 15: 53$ & 4.4 & 6.3 \\
\hline average & 01:29:04 & $+67: 15: 33$ & 3.5 & 4.4 \\
\hline \multicolumn{5}{|c|}{ Candidate remnant coordinates } \\
\hline $\mathrm{Pa} 30$ & 00:53:11.2 & $+67: 30: 02.4$ & & \\
\hline $3 \mathrm{C} 58$ & 02:05:37 & $+64: 49: 42$ & & \\
\hline
\end{tabular}

Note. The original SN 1181 coordinates (e.g., 1:30, +65) have been precessed from B1950 to J2000. The uncertainty in these values remains high. The last two columns show the distance to the two candidate remnants in degrees. References to the historical positions are listed in Hoffmann et al. (2020).

remained visible for 185 days. Given that the limit of naked-eye visibility is $m \approx 5.5$, this indicates $4.5-6$ mag of fading over this period. The long period of naked-eye visibility is typical for a supernova but does not fit a typical nova duration of days to a few weeks.
Pa 30 is a source of diffuse X-ray emission with a spatial extent in serendipitous Swift XRT and pointed XMM-Newton EPIC observations larger than that in the mid-IR WISE and optical [O III] images (Figure 1). The XMM-Newton EPIC X-ray spectra of both the CS and nebula have been fitted with plasma emission models with significant enrichment in neon, magnesium, silicon, and sulfur (Oskinova et al. 2020), which has been interpreted as the result of the incomplete carbon and oxygen fusion expected in Type Ia SNe.

$\mathrm{Pa} 30$ can accordingly be classified as a supernova remnant (Gvaramadze et al. 2019; Oskinova et al. 2020), but the bright CS, which lacks hydrogen and helium, shows it is not a common type. Instead it points at a Type Iax supernova as suggested by Oskinova et al. (2020), that is, a subluminous SN Ia event in which the star does not self-destruct. While the exact mechanisms leading to a Type Iax SN are still not fully understood, they are believed to arise from either the failed detonation of a carbon-oxygen (CO) WD accreting material from a helium donor star (single-degenerate scenario; e.g., Jordan et al. 2012; Kromer et al. 2015) or from a CO WD merging with a heavier oxygen-neon $(\mathrm{ONe}) \mathrm{WD}$ (doubledegenerate scenario; Kashyap et al. 2018) where the accretion disk itself deflagrates.

A key discriminant between typical $\mathrm{SNe}$ of Type Ia and Type Iax is the expansion velocity with $\sim 10,000 \mathrm{~km} \mathrm{~s}^{-1}$ for 


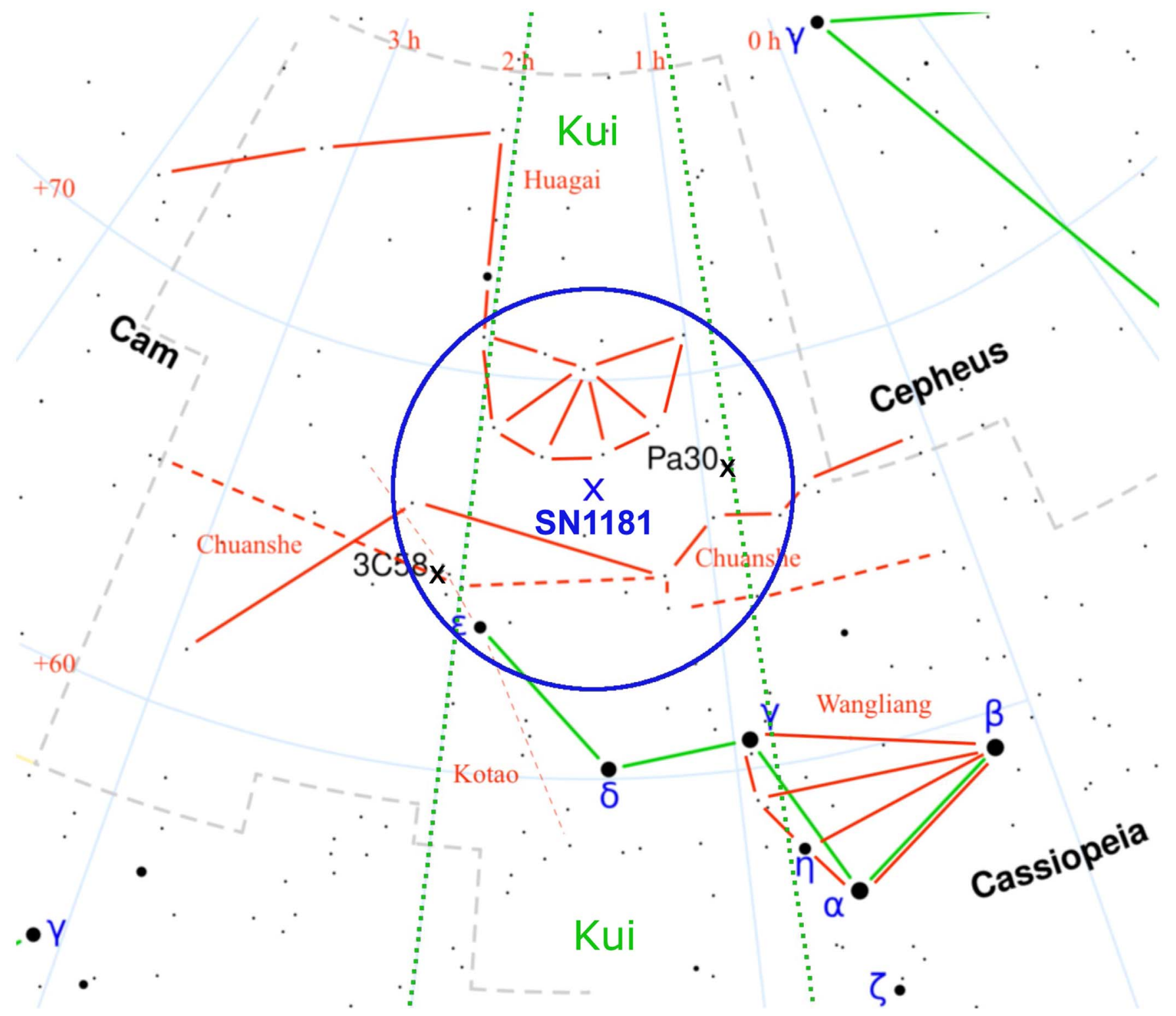

Figure 3. The region of SN 1181, as described in Hsi (1957) with the asterisms according to Hoffmann et al. (2020, their Figure A5) and Stephenson \& Green (2002, their Figure 9.1, red dashed line shows their asterism for Chuanshe) indicated. Pa 30 and 3C 58 are indicated by black crosses. The green line indicates Cassiopeia. The Chinese "lunar lodge" Kui is between the two green dotted lines. The supernova was stated to lie between Huagai and Chuanshe, near Wangliang. The best estimated average position of SN 1181 is given by a blue cross surrounded by a blue error circle of radius $5^{\circ}$. The background star chart was created using PP3Celestial Chart Generation (http://pp3.sourceforge.net/).

Type Ia and currently only $2000-7000 \mathrm{~km} \mathrm{~s}^{-1}$ for the much smaller known population Type Iax (Jha 2017). Our measured expansion velocity for the nebula is $1100 \mathrm{~km} \mathrm{~s}^{-1}$, which clearly disfavors typical Type Ia. The observed value for SN1181 is lower than the currently lowest expansion velocity of known Type Iax, but given the small sample size with measured expansion velocities the full typical range for this class is not well constrained. Even so the result still clearly favors a Type Iax event. Type Iax SNe also show a large range of peak magnitudes, which at the faint end $(-13$ to -14 mag; Jha 2017) overlap with the likely magnitude for SN 1181 at a distance of $2.3 \mathrm{Kpc}$. This also independently supports this classification.

Based on the current stellar luminosity, Pa 30 is likely a double-degenerate merger (Gvaramadze et al. 2019) where Oskinova et al. (2020) interpret it as an ONe-CO WD merger based on the nebula's neon abundance. Models for high-mass ONe-CO mergers (Kashyap et al. 2018) predict a faint SN Iax with an absolute magnitude of only $-11.3 \mathrm{mag}$. This is a bit fainter than what we find for SN 1181 but indicates that the Oskinova et al. (2020) interpretation may be supported by the faint magnitude of the event.

\section{Methods}

\subsection{Coordinates}

The likely position of SN 1181 based on the historical descriptions is reported by Hoffmann et al. (2020) as R.A.: 01:31:14 decl.: $+70: 15: 50$ (J2000) with an uncertainty of $\sim 4^{\circ}$ (their Table 5). They also report previous determinations by four other authors. We averaged all five positions, converted to 
J2000 coordinates. This gives R.A.: 01:29:04 decl.: +67:15:33 . The uncertainty on the position is taken as $5^{\circ}$.

The proposed coordinates of SN 1181 (accurate to $5^{\circ}$ ) and the two candidate remnants are listed in Table 1.

Following Hsi (1957) and Hoffmann et al. (2020), we have compared the locations of both candidates to the original Chinese and Japanese reports described by Stephenson \& Green (2002). Two texts from China place the guest star "in the "Kui lunar lodge," trespassing against Chuanshe and guarding the 5th star of Chuanshe" (South China) and "in Huagai" (North China). A record from Japan describes it "close to Wangliang and guarding Chuanshe." The Kui lunar lodge and the three asterisms are depicted in Figure 3, which is based on Figure A5 of Hoffmann et al. (2020, we note that the decl. scale in their Figure A5 is incorrect). Wangliang contains the brightest stars in the area, which are part of the modern constellation Cassiopeia. The seven stars of Huagai, although much fainter, form a well-defined cluster shaped like a parasol and are also fairly easily identified. The exact position of Chuanshe is less certain. This asterism consists of nine dim stars barely visible to the unaided eye. Two possible positions (Hoffmann et al. 2020, their Figure A5; Stephenson \& Green 2002, their Figure 9.1) are shown in Figure 3. Taken together, the supernova can be expected to have occurred in between Huagai and Chuanshe. Our averaged best estimate of its position is given by a blue cross centered on a blue error circle of radius $5^{\circ}$. It is notable that none of the records mention the asterism Kotao (thin dashed line in Figure 3), although it crosses Chuanshe at the location of $3 \mathrm{C} 58$. One would also expect that the location of 3C 58 would have been better described as being close to $\varepsilon$ Cas, a bright third magnitude star. In contrast, the location of Pa 30, also denoted by a labeled black cross, fits the description well and is in better agreement with its association with Huagai. However, the descriptions are not accurate enough to decide between the two candidates on positional information alone though $\mathrm{Pa} 30$ is clearly favored.

\subsection{Observations}

We obtained optical spectroscopy of both the star and the nebula on 2016 July 8 using long-slit observations with the OSIRIS instrument of the $10 \mathrm{~m}$ GTC telescope (Cepa 2010) using the grism R1000B. The 7!!4 slit with a width of 0 !" 8 was placed at a position angle $30^{\circ}$ east of north, slightly off-center from the central star to reduce contamination from a bright field star. Total exposure time was $2 \times 20$ minutes. A factor $2 \times 2$ binning was used that provides a spatial scale of 0 ". 254 pixel $^{-1}$ (with a seeing of 1 !' 35 ) and a spectral resolution of about $2 \AA$ $(R=1000)$. The wavelength coverage was $3700-7000 \AA$. We used the standard, well-constrained GTCMOS pipeline for the data reduction.

On 2014 October 15 the DSH team used the SparsePak integral field unit (IFU) of the Bench mounted spectrograph on the $3.5 \mathrm{~m}$ WIYN telescope at KPNO to observe Pa 30 as a planetary nebula candidate. They did not see the expected emission lines and did not extract the central star's spectrum. The exposure time was $2 \times 20$ minutes. The IFU mode offers 82 fibers of $4 . " 7$ diameter in a $72^{\prime \prime} \times 71^{\prime \prime}$ grid (Bershady et al. $2004)$. The wavelength coverage with grating $600 \mathrm{~V}\left(10^{\circ} .1\right.$ Littrow blaze angle) was 4280-7095 A giving a spectral resolution of $3.35 \AA$. We re-reduced this archived data using the well-constrained pipeline of Ritter \& Washuettl (2004) and standard, reliable IRAF packages extracting both the star and nebula spectra. Since the dedicated sky fibers were also placed

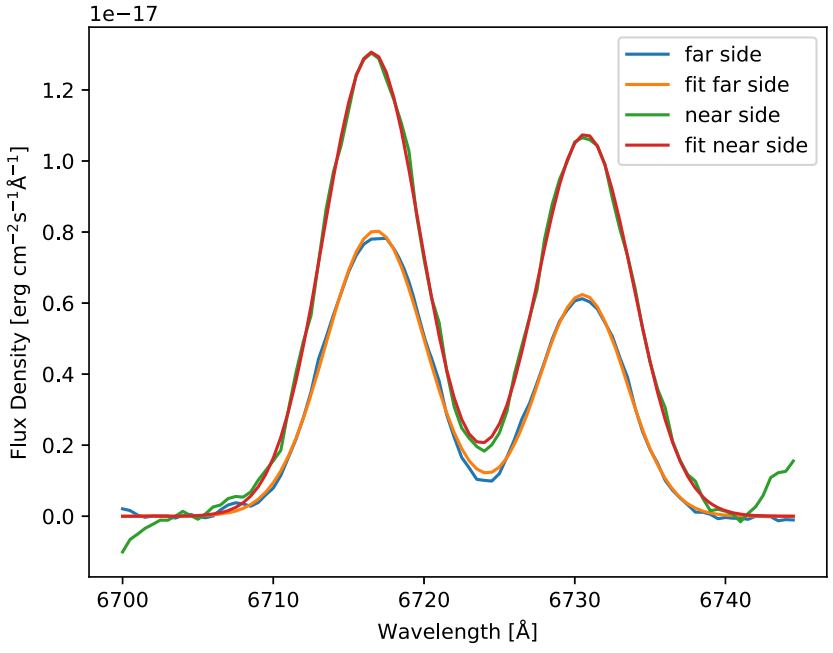

Figure 4. Sum of the background-subtracted and radial-velocity corrected [S II] doublets for the near side and the far side together with the fitted double Gaussians.

on regions with potential nebular emission, we subtracted the sky using standard principal component analysis.

\subsection{Expansion Velocity Measurement}

For the measurement of the expansion velocity of Pa 30 we used the long-slit GTC/OSIRIS spectrum. The data reduction was done using the standard and reliable GTCMOS pipeline which for the setup described above gives a very reliable wavelength solution and a radial-velocity accuracy of a few $\mathrm{km} \mathrm{s}^{-1}$. This was confirmed in our data. For each spectral column the two sets of [S II]-doublet features, arising as a result of the fast expansion, were identified and their radial velocities measured using standard cross-correlation with an artificial spectrum at zero velocity. Standard goodness-of-fit tests for the resulting chi-square functions and Gaussian fits of their respective minima were performed to remove bad identifications. Figure 4 shows the sum of the background-subtracted, radial-velocity corrected [S II] doublets for the positive (near side) and negative (far side) radial velocities, together with their corresponding double-Gaussian fits.

\subsection{Interstellar Extinction}

The neutral hydrogen column density estimates (HI4PI Collaboration et al. 2016) yield an extinction of $A_{V}=2.24 \pm 0.1$ mag. The higher-resolution 3D IPHAS extinction map (Sale et al. 2014) indicates a higher extinction of $A_{0}=2.52 \pm 0.40 \mathrm{mag}$ (monochromatic).

We investigated 3D extinction maps based on the Gaia-2MASS (Lallement et al. 2019) and Gaia-PanSTARRS-2MASS (Green et al. 2019) databases. In the first case (Lallement et al. 2019), we find a color excess $E(B-V)=0.87$ and therefore $A_{V}=2.7 \mathrm{mag}$. In the latter case (Green et al. 2019), $E(g-r)=0.70$ was found at $2.3 \mathrm{kpc} . E(B-V) / E(g-r)=0.884$ to 0.996 ; therefore, $A_{V}$ could be within the range 1.92-2.17 mag.

We also selected stars within $10^{\prime}$ of Pa 30 in the Gaia EDR3 catalog with parallax uncertainty $\sigma_{\pi} / \pi<0.5$ with a nonzero $A_{G}$ from Gaia DR2. The median $A_{G}$ is about $2.25 \mathrm{mag}$. Using Table 13 of Jordi et al. (2010), we extrapolate $A_{G} / A_{V}$ ratios between 0.88 and 1.02 for stars hotter than $50 \mathrm{kK}$, giving an interstellar extinction of $A_{V}=2.4 \pm 0.2$ that we have adopted for dereddening. 


\section{Conclusions}

The previous association of 3C 58 with SN 1181 was based in part on the lack of another viable candidate, but it has difficulties with the discrepant ages from the observed expansion velocity, proper motion of the knots, neutron star cooling models, and pulsar spin-down rate (Bietenholz 2006; Fesen et al. 2008). Kothes (2013) proposed a much smaller distance to $3 \mathrm{C} 58$ than previously found $(2 \mathrm{kpc}$ versus $3.2 \mathrm{kpc}$; Roberts et al. 1993), which reduces the age discrepancy from the radial velocity and the angular size, but this would then imply an event with a very low absolute peak brightness of between -13.4 and -14.3 mag (for the assumed apparent peak brightness of 0 mag in that paper). If 3C 58 was the counterpart of SN 1181, this would make it one of the faintest SNe ever recorded. Pa 30 now provides an excellent, viable candidate for the SN 1181 eruption that fits the location, the age, the brightness, and even the visible duration given its likely Type Iax nature.

We therefore conclude that $\mathrm{Pa} 30$ is the remnant of the SN 1181 supernova. SN 1181 was until now the only remaining historical supernova of the last millennium without a certain counterpart. It is also the first recorded supernova Type Iax event in the Galaxy of which there are now perhaps fully two cases known (Zhou et al. 2021) but is the only one where detailed studies of the remnant star and nebula are possible, and for which the doubledegenerate merger scenario has strong observational support (Gvaramadze et al. 2019; Oskinova et al. 2020). Given the extreme nature of Parker's star itself (Gvaramadze et al. 2019; Oskinova et al. 2020) and our linking it to the $1181 \mathrm{AD}$ supernova, this source is of considerable scientific and historical interest. Parker's star is the only Wolf-Rayet star known that is neither the result of a massive Pop I progenitor nor the central star of a planetary nebula, but is the result of an $\mathrm{ONe}-\mathrm{CO}$ WD merger that accompanied a Type Iax supernova explosion that now has an historical basis.

We acknowledge with thanks Matthias Kronberger and Dana Patchick of the Deep Sky Hunters amateur group for their dedication in discovering new PNe and uncovering Pa 30. We also thank Dr. Laurence Sabin (UNAM) for designing the GTC/ OSIRIS observations on behalf of other team members Q.A.P. and A.A.Z. Q.A.P. thanks the Hong Kong Research Grants Council for GRF research support under grants 17326116 and 17300417. A.R. and F.L. thank HKU for the provision of postdoctoral fellowships under Q.A.P., while F.L. also acknowledges funding from MTA Hungary (OTKA project No. K-132406). A.A.Z. thanks the Hung Hing Ying Foundation for the provision of a visiting professorship at HKU and acknowledges funding from the UK STFC under grant ST/T000414/1. M.A.G. was funded under grant number PGC2018-102184-B-I00 of the Ministerio de Educación, Innovación y Universidades cofunded with FEDER funds. Finally, we thank Ms. Elizabeth Yang of Hong Kong for help with sourcing and understanding the ancient Chinese records of this event.

This work made use of the University of Hong Kong/ Australian Astronomical Observatory/Strasbourg Observatory H-alpha Planetary Nebula (HASH PN) database, hosted by the Laboratory for Space Research at the University of Hong Kong; the extinction map query page, hosted by the Centre for Astrophysics and Planetary Science at the University of Kent; and of data products from the Wide-field Infrared Survey Explorer, which is a joint project of the University of California, Los Angeles, and the Jet Propulsion Laboratory/ California Institute of Technology, funded by the National Aeronautics and Space Administration. Based on observations obtained with XMM-Newton, an ESA science mission with instruments and contributions directly funded by ESA Member States and NASA.

Facilities: WISE, GALEX (near-UV), XMM (Newton), GTC:10.0 m, OSIRIS, KPNO:WIYN3.5 m, SparsePak, KPNO:2.1 m.

Software: astropy (Astropy Collaboration et al. 2013, 2018), PP3 - Celestial Chart Generation (http://pp3.sourceforge.net/) created by Torsten Bronger, Inkscape (https://inkscape.org/) that is Free and Open Source Software licensed under the GPL.

\section{ORCID iDs}

Andreas Ritter (1) https://orcid.org/0000-0003-0869-4847 Quentin A. Parker (10 https://orcid.org/0000-0002-2062-0173 Foteini Lykou (1) https://orcid.org/0000-0002-6394-8013 Albert A. Zijlstra (1) https://orcid.org/0000-0002-3171-5469 Martín A. Guerrero (1) https://orcid.org/0000-0002-7759-106X Pascal Le Dû (i) https://orcid.org/0000-0003-2385-0967

\section{References}

Astropy Collaboration, Price-Whelan, A. M., Sipőcz, B. M., et al. 2018, AJ, 156,123

Astropy Collaboration, Robitaille, T. P., Tollerud, E. J., et al. 2013, A\&A, 558, A33

Bailer-Jones, C. A. L., Rybizki, J., Fouesneau, M., Demleitner, M., \& Andrae, R. 2021, AJ, 161, 147

Bershady, M. A., Andersen, D. R., Harker, J., Ramsey, L. W., \& Verheijen, M. A. W. 2004, PASP, 116, 565

Bietenholz, M. F. 2006, ApJ, 645, 1180

Cepa, J. 2010, ASSP, 14, 15

Chevalier, R. A. 2004, AdSpR, 33, 456

Cutri, R. M., Wright, E. L., Conrow, T., et al. 2012, yCat, 2311

Fesen, R., Rudie, G., Hurford, A., \& Soto, A. 2008, ApJS, 174, 379

Green, D. A. 2002, HiA, 12, 350

Green, G. M., Schlafly, E. F., Zucker, C., Speagle, J. S., \& Finkbeiner, D. P. 2019, ApJ, 887, 93

Gvaramadze, V. V., Gräfener, G., Langer, N., et al. 2019, Natur, 569, 684

HI4PI Collaboration, Ben Bekhti, N., Flöer, L., et al. 2016, A\&A, 594, A116

Hoffmann, S. M., Vogt, N., \& Protte, P. 2020, AN, 341, 79

Hsi, T.-T. 1957, SCoA, 2, 109

Jha, S. W. 2017, Type Iax Supernovae (Berlin: Springer), 375

Jordan, G. C. I., Perets, H. B., Fisher, R. T., \& van Rossum, D. R. 2012, ApJL, 761, L23

Jordi, C., Gebran, M., Carrasco, J. M., et al. 2010, A\&A, 523, A48

Kashyap, R., Haque, T., Lorén-Aguilar, P., García-Berro, E., \& Fisher, R. 2018, ApJ, 869, 140

Kothes, R. 2013, A\&A, 560, A18

Kromer, M., Ohlmann, S. T., Pakmor, R., et al. 2015, MNRAS, 450, 3045

Kronberger, M., Parker, Q. A., Jacoby, G. H., et al. 2016, JPhCS, 728, 072012

Lallement, R., Babusiaux, C., Vergely, J. L., et al. 2019, A\&A, 625, A135

Oskinova, L. M., Gvaramadze, V. V., Gräfener, G., Langer, N., \& Todt, H. 2020, A\&A, 644, L8

Parker, Q. A., Bojičić, I. S., \& Frew, D. J. 2016, JPhCS, 728, 032008

Ritter, A., \& Washuettl, A. 2004, AN, 325, 663

Roberts, D. A., Goss, W. M., Kalberla, P. M. W., Herbstmeier, U., \& Schwarz, U. J. 1993, A\&A, 274, 427

Sale, S. E., Drew, J. E., Barentsen, G., et al. 2014, MNRAS, 443, 2907

Stephenson, F., \& Green, D. 2002, Historical Supernovae and Their Remnants (Oxford: Oxford Univ. Press)

Stephenson, F. R., \& Green, D. A. 1999, A\&G, 40, 27

Zhou, P., Leung, S.-C., Li, Z., et al. 2021, ApJ, 908, 31 